\title{
Infrared radiation detector interrogated by optical frequency-domain reflectometer
}

\author{
Kivilcim Yüksel $^{\mathrm{a}, *}$, Christophe Caucheteur ${ }^{\mathrm{a}}$, Jean-Michel Renoirt ${ }^{\mathrm{b}}$, Marc Debliquy ${ }^{\mathrm{b}}$, \\ Patrice Mégret ${ }^{a}$, Marc Wuilpart ${ }^{a}$ \\ ${ }^{a}$ Electromagnetism and Telecommunication Department, Faculté Polytechnique, U-MONS, Boulevard Dolez 31, 7000 Mons, Belgium \\ ${ }^{\mathrm{b}}$ Material Science Unit, Faculté Polytechnique, U-MONS, Belgium
}

\section{A R T I C L E I N F O}

\section{Article history:}

Received 22 September 2011

Received in revised form

4 November 2011

Accepted 18 November 2011

Available online 6 December 2011

Keywords:

Fiber optics sensors

Fiber Bragg Gratings

Fiber measurements

Optical frequency domain reflectometer

\begin{abstract}
A B S T R A C T
We experimentally demonstrated a fast infrared (IR) radiation sensor. It is capable of measuring IR radiation independently from the environmental temperature fluctuations. Experimental work shows that this IR detection prototype have strong conveniences (fast response and reliability in harsh environment) compared to previous detectors which makes it a very good option for early fire detection systems.
\end{abstract}

(c) 2011 Elsevier Ltd. All rights reserved.

\section{Introduction}

Among the fiber sensors using the Fiber Bragg Gratings (FBGs) as sensing points, a large variety of new applications can be envisaged resulting from the association of the FBGs with a sensitive layer. Examples include humidity sensing [1], $\mathrm{pH}$ monitoring [2], and gas sensing such as hydrogen [3,4] or methane [5]. Infrared (IR) radiation detector is another example of this group of applications and presents a very strong potential for early fire detection.

An IR radiation detector using a pair of FBGs has been recently demonstrated [6,7]. This method is called fibered bolometer as it implements the operating principle of a standard bolometer and is schematically represented in Fig. 1.

It works as follows: one of the FBGs in the pair is covered with an IR-absorbing material while the other is protected from the radiation in order to be used as a temperature reference. The useful signal is the temperature increase of the coated FBG when exposed to IR radiation compared to the reference FBG. The temperature change is determined either by measuring the shift in the Bragg wavelengths using an optical spectrum analyser (OSA) [6], or by measuring the total reflected power from the FBG pair using a powermeter [7]. In order to have the quasi-distributed capability, both of these approaches require the wavelength

\footnotetext{
* Corresponding author.

E-mail address: kivilcim.yuksel@umons.ac.be (K. Yüksel).
}

demultiplexed interrogation which complicates the sensor design. Moreover OSA is a slow and expensive device to be used as an interrogator tool. In the latter approach (the use of powermeter), the useful information is encoded in the total reflected power from the FBG pair which means that the operating zone is limited to a certain IR flux value that is linked to the FBGs spectral bandwidth.

Optical frequency domain reflectometry (OFDR) is a technique for high-resolution metrology which has been attracting great attention as an interrogating tool in both optical fiber sensors [8] and telecommunication networks [9]. In a basic configuration of an OFDR, the optical frequency of a tunable laser source (TLS) is swept linearly and phase continuously in time. Then, the frequency-modulated optical signal (probe signal) is split into two paths, one of which probes the device under test (DUT) whereas the other is used as reference signal (or local oscillator). The reference signal returning from the reference mirror and test signal returning from the reflection sites in the test arm coherently interferes at the coupler. Superposition of the interfering signals is converted into electrical domain by the detector which yields the beat terms that are related to the optical amplitude and phase responses of the reflection sites in the DUT. In order to observe these beat terms, the photocurrent at the detector output is sampled and Fourier transformed into frequency domain where the beat terms appear as peaks.

In this paper, we report an IR detection sensor using fibered bolometers as sensing points and interrogated by OFDR. The main advantage of our system compared to previous work is that the 


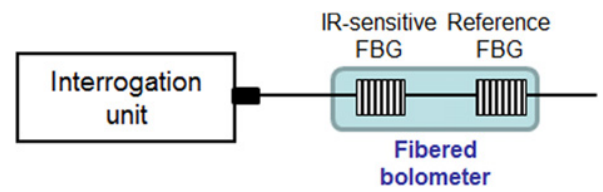

Fig. 1. Schematic representation of the fibered bolometer.

information is encoded in the Bragg wavelength shift (which is not affected by unwanted power fluctuations) and that it is possible to interrogate, within a few seconds, a cascade of FBGpairs with identical spectral characteristics to obtain a quasidistributed sensor configuration. A wide panel of applications can be envisaged such as early fire detection and monitoring of hot spots. For the fire detection application our solution would be preferable over the conventional smoke and dust detectors as they are influenced from environmental conditions for certain applications (e.g. combustion gas influences the conventional detectors in road traffic tunnels [10]).

\section{Sensing principle}

In order to evaluate the feasibility of the proposed sensor, one should first analyse the properties of absorption spectra of the sensor together with the emission spectra of the source. The major part of the IR radiations emitted by hot spots covers the range from $0.8 \mu \mathrm{m}$ to $15 \mu \mathrm{m}$. The emission spectrum of a heat source at different temperatures can be explained by the Planck's law which describes the spectral radiance emitted from a black body at different temperatures. Planck's law is written as

$I_{\lambda, T}=\frac{2 h c^{2}}{\lambda^{5}} \frac{1}{\exp ^{(h c / \lambda k T)-1}}$

where $I_{\lambda, T}$ is the emitted power intensity (emitted power per unit area of emitting surface in the normal direction, per unit solid angle, for a given wavelength and temperature), $h$ is the Planck's constant $\left(6.62 \times 10^{-34} \mathrm{~J} \mathrm{~s}\right), c$ is the speed of the light, $k$ is Boltzmann's constant $\left(1.38 \times 10^{-23} \mathrm{~J} / \mathrm{K}\right)$ and $T$ the temperature of the black body. The normalised spectral radiance intensity computed by (1) at different temperatures is shown in Fig. 2. It can be seen from this figure that the higher the temperature the narrower the distribution centered on a lower wavelength.

In a flamming fire, the temperature is about $1500 \mathrm{~K}\left(1227^{\circ} \mathrm{C}\right)$ which means that most important part of the IR spectrum radiates in the wavelength range below $4 \mu \mathrm{m}$ (see Fig. 2).

IR spectrum radiating in the wavelength range below $5 \mu \mathrm{m}$ is not absorbed by silica $\left(\mathrm{SiO}_{2}\right)$ [6]. Therefore, a bare FBG has got a weak response to IR radiation in this wavelength region and should be covered with an IR-absorbing material. Using a thin layer of copper(II) oxide (CuO) was proved to be efficient in increasing the absorption efficiency [6]. Fig. 3 compares the transmittance spectra of $\mathrm{SiO}_{2}(1 \mathrm{~mm}$ thickness) and $\mathrm{CuO}(44 \mu \mathrm{m}$ thickness). $\mathrm{CuO}$ yields a strong absorption for wavelengths below $5 \mu \mathrm{m}$ is used for the coated FBG (association of $\mathrm{SiO}_{2}$ and $\mathrm{CuO}$ ) with the good response to detect hot spots. The coated FBG converts the radiation into heat. Therefore, when subject to IR radiations, the temperature of the FBG under exposure will increase and its Bragg wavelength will be shifted by $\Delta \lambda$.

The efficiency of the sensor can be further improved by putting a thin glass tube (a capillary tubing) on the CuO-coated FBG. Greenhouse effect is the physical mechanism behind this efficiency increase and can be explained as follows [6]: radiations below $4 \mu \mathrm{m}$ go through the glass tube and are absorbed by the $\mathrm{CuO}$ layer. Longer wavelengths are re-radiated in all directions and are confined in the glass tube as glass absorbs radiations

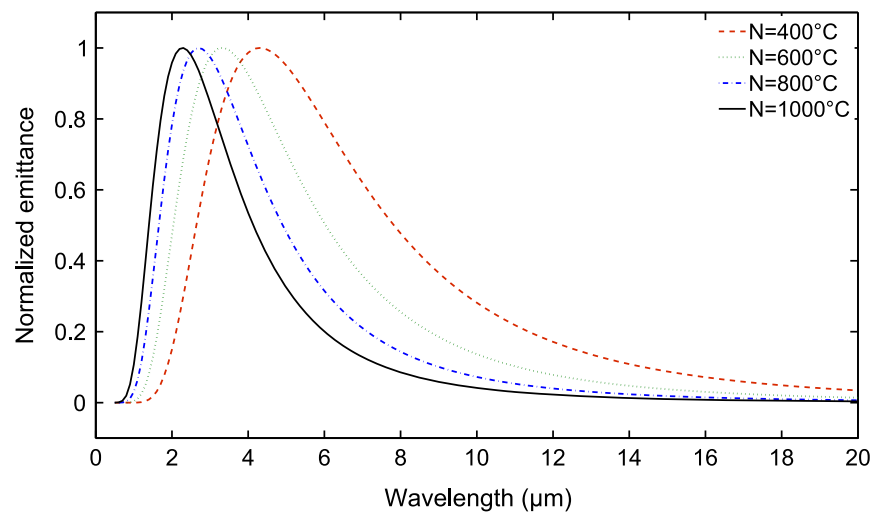

Fig. 2. Spectral radiance of a heat source at different temperatures.

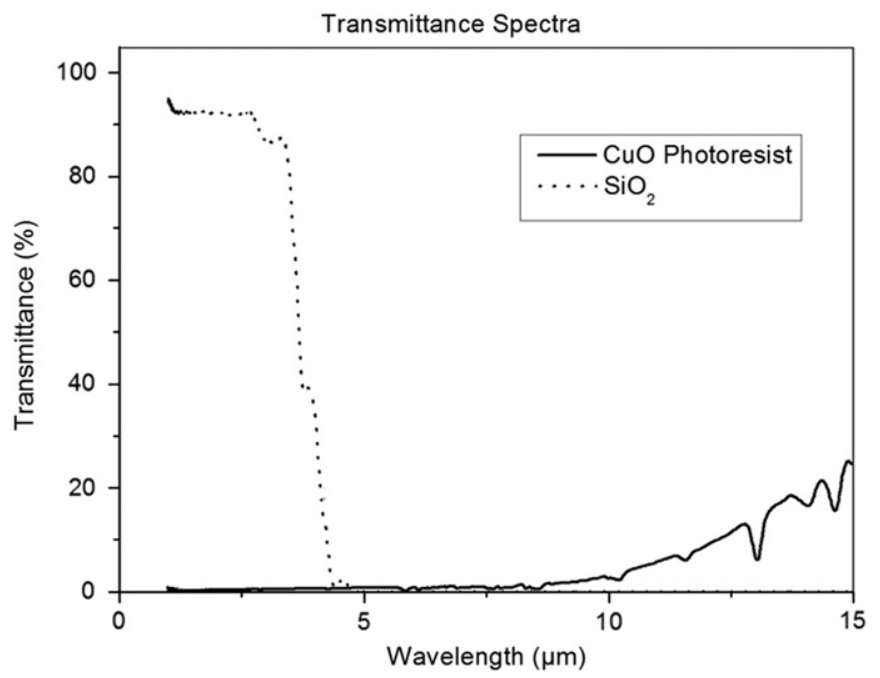

Fig. 3. Transmittance spectra of $\mathrm{SiO}_{2}$ and $\mathrm{CuO}$.

above $4 \mu \mathrm{m}$. Then, the glass tube re-transfers energy to the grating by radiation process and increases the fiber temperature for a given radiation flux. The capillary tube offers a larger surface to collect the radiations that also increases the sensor efficiency.

It was experimentally shown that the sensitivity can be improved for a given temperature from about $0.008 \mathrm{pm} /\left(\mathrm{W} / \mathrm{m}^{2}\right)$ to $0.04 \mathrm{pm} /\left(\mathrm{W} / \mathrm{m}^{2}\right)$ by using a capillary tubing on the grating [6].

The coated FBG together with the reference FBG composing the fibered bolometer is used as sensing element. Each FBG pair (bolometer) can be placed in a metal tube (e.g. aluminum) to concentrate the incident radiations on the coated FBG and to shield the reference FBG from IR radiations. The packaging made of a metal tube and the coated FBG inside the capillary (glass) tubing is shown in Fig. 4. Parabolic shape is preferable (the FBG is placed in the focal point) for the packaging to enhance the concentration of radiations on the sensor.

In a quasi-distributed configuration, many of these sensing elements can be cascaded. Operation principle of the quasidistributed IR sensor interrogated by OFDR is schematically represented in Fig. 5.

The OFDR unit launches into the network a frequency-modulated continuous-wave (CW) signal and measures the interference signals created by the FBGs (reference and coated). Each FBG creates a periodical beat signal (i.e. a reflection peak on the OFDR trace) with a unique beat frequency. One of each FBG-pair is coated with an IR-absorbing layer which converts the radiation into heat. Therefore, when subject to IR radiations, the temperature of the 


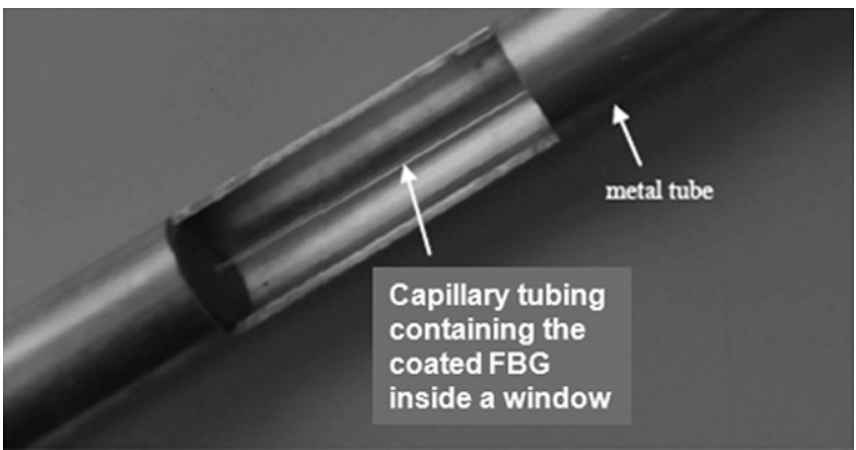

Fig. 4. Packaging made of a metal tube and the coated FBG inside the capillary tubing.

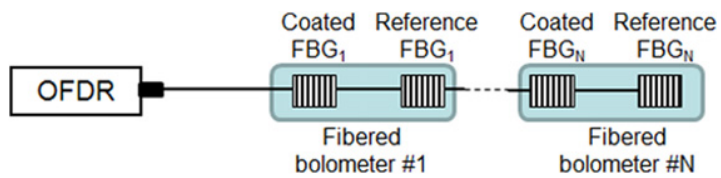

Fig. 5. Principles of the interrogating system based on OFDR and cascaded matched FBG-pairs as sensing elements.

FBG under exposure will increase and its Bragg wavelength will be shifted by $\Delta \lambda$. As the information on the physical parameter is found in the wavelength shift, the goal of the interrogating system is to retrieve the Bragg wavelength of the coated grating. For this purpose, reflection spectrum of each coated FBG is demodulated by band-pass filtering the OFDR trace around the related beat frequency with sufficient bandwidth. Then, an inverse fast Fourier transform (IFFT) applied on this selected portion can be used to recover the complex reflection spectrum of each grating in the cascade independently from others as explained in [8,11]. Finally, from the estimation of their Bragg wavelength and from their calibration characteristics, the IR radiation flux is deduced for each coated FBG in the sensor. Reference FBG is used to compensate the effect of temperature changes. A proprietary OFDR set-up was used in the experiments [8].

\section{Experimental results}

Proof of concept experiments was realized on a single matched FBG pair. The coated and reference FBGs are separated by $45 \mathrm{~cm}$.

The gratings used in the sensor were manufactured with hydrogenated standard germanium-doped silica single mode fiber in the Mons University clean room facilities by means of a continuous-wave frequency-doubled argon-ion laser emitting at $244 \mathrm{~nm}$ through a 24-mm-long apodized phase mask yielding FBGs centered around $1534.5 \mathrm{~nm}$. CuO (copper oxide) was deposited (a layer of few tens of microns) on one FBG of the pair by polymeric encapsulation method that includes the following steps [6]:

- dispersion of $\mathrm{CuO}$ powder in a UV sensitive polymer,

- mixing the $\mathrm{CuO}$ powder and the polymer for several minutes,

- exposure of the suspension to ultrasounds during $30 \mathrm{~min}$,

- putting the fiber section around the FBG into this suspension,

- drying the FBG at $110^{\circ} \mathrm{C}$,

- UV-curing for a few seconds.

To further improve the sensitivity, the coated FBG is centered into glass capillary tube and both FBGs are packaged inside an aluminum tube as explained before.
The OFDR is operated over $3 \mathrm{~nm}$ sweep span around $1534.5 \mathrm{~nm}$. Sweep time is $300 \mathrm{~ms}$. The output power of OFDR is $3.9 \mathrm{~mW}$. The applied IR radiation flux is modified by changing the distance between the coated FBG and the IR source. The results are checked by using a radiant flux sensor (Captec, sensitivity: $0.438 \mu \mathrm{V} / \mathrm{m}^{2}$, active area: $10 \times 10 \mathrm{~mm}^{2}$ ) which is placed near to the coated FBG as represented in Fig. 6 . The voltage signal at the output of this electronic flux sensor is measured by an high accuracy multimeter (see Fig. 6).

Figs. 7 and 8 represent the demodulated reflection spectrum of the coated FBG and of the reference FBG for different IR radiations. The shape modification of the reference FBG spectrum when there is no flux is attributed to the fact that a small portion of the spectrum is masked by the coated FBG (see Fig. 6).

One can clearly observe in Fig. 9 the linear correlation between the shift in the demodulated Bragg wavelength of the coated FBG and the incident flux intensity measured by the electronic radiant flux sensor. The slope computed on the linear fit is $0.07 \mathrm{pm} /(\mathrm{W} /$ $\mathrm{m}^{2}$ ) which has a good agreement with the previous results reported in $[7,6]$.

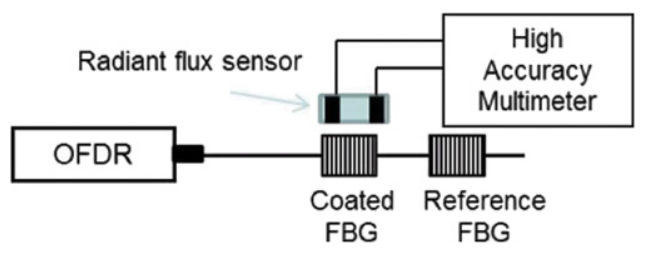

Fig. 6. Experimental set-up.

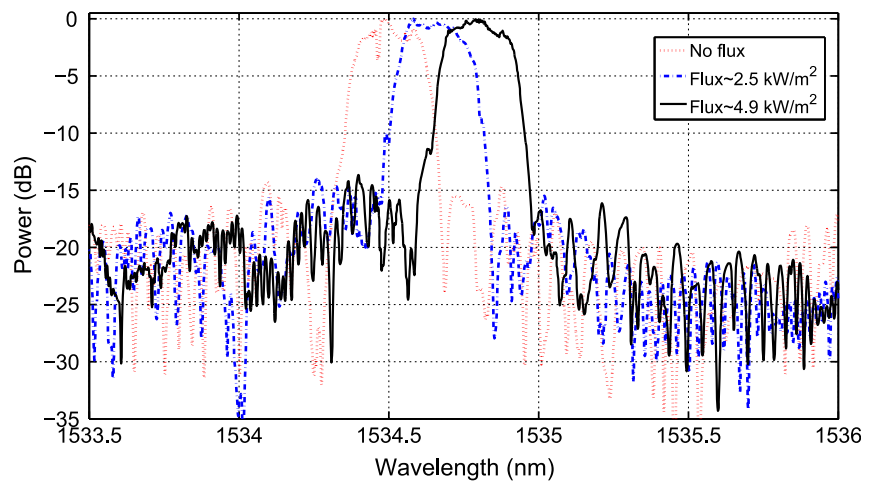

Fig. 7. Demodulated reflection spectrum of the coated FBG for different IR radiations.

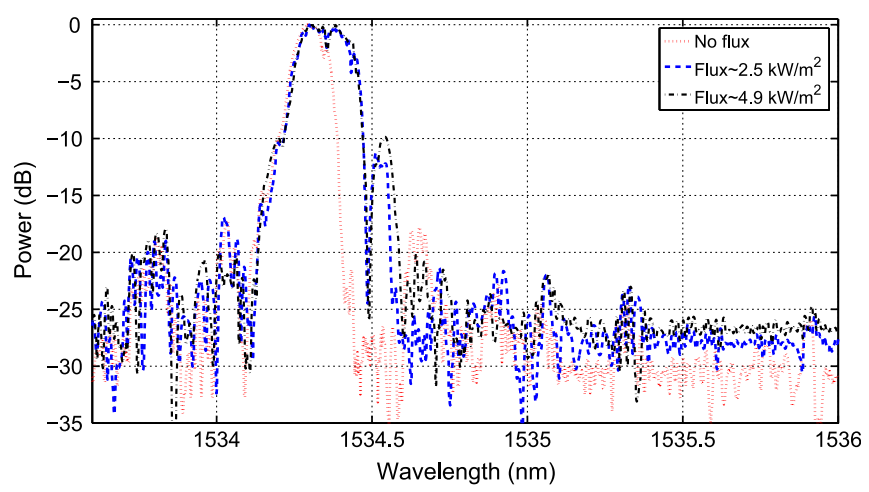

Fig. 8. Demodulated reflection spectrum of the reference FBG for different IR radiations. 


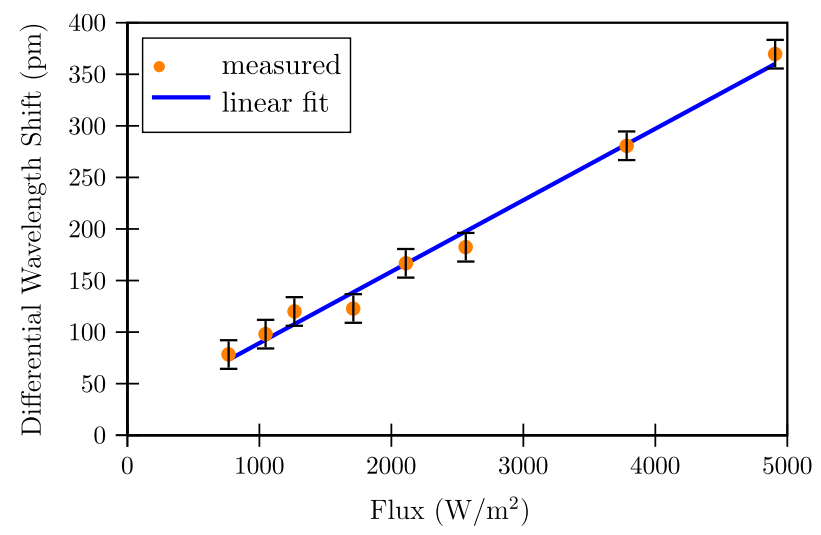

Fig. 9. Evolution of demodulated Bragg wavelength as a function of incident flux intensity.

In the last part of the measurements, for a given IR flux value applied on the coated FBG, the measurements were repeated ten times. These repeatability measurements highlighted a standard deviation on the demodulated Bragg wavelength of $15 \mathrm{pm}$ (which corresponds to a flux value of about $215 \mathrm{~W} / \mathrm{m}^{2}$ ).

\section{Conclusions}

We experimentally demonstrated a fast (interrogation time is a few seconds) and simple technique for infrared detection. The main advantage of the proposed method is that several low reflective FBG pairs can be cascaded in a quasi-distributed scheme and can be interrogated in a few seconds.

A wide panel of applications can be envisaged such as early fire detection and monitoring of hot spots. In the context of fire detection, smoke and dust detectors may not be suitable for certain applications, for instance, in road traffic tunnels where combustion gas influences the detector [10]. IR emission sensor is advantageous over conventional systems thanks to its fast response and reliability in harsh environment.

\section{Acknowledgments}

This research was supported by Fonds de la Recherche Scientifique-Crédit aux Chercheurs (FNRS) and the Interuniversity Attraction Pole IAP 6/10 program of the Belgian Science Policy. Authors acknowledge the financial support of the project $O p t i^{2}$ Mat by the Walloon Region.

\section{References}

[1] Kronenberg P, Rastogi PK, Giaccari P, Limberger HG. Relative humidity sensor with optical fiber Bragg gratings. Opt Lett 2002;27:1385-7.

[2] Corres JM, del Villar I, Matias IR, Arregui FJ. Fiber-optic pH-sensor in longperiod fiber gratings using electrostatic self-assembly. Opt Lett 2007;32: 29-31.

[3] Buric M, Chen KP, Bhattarai M, Swinehart PR, Maklad M. Active fiber Bragg grating hydrogen sensors for all-temperature operation. IEEE Photonics Technol Lett 2007;19:255-7.

[4] Caucheteur C, Debliquy M, Lahem D, Mégret P. Hybrid fiber gratings coated with a catalytic sensitive layer for hydrogen sensing in air. Opt Express 2008;16:16854-9.

[5] Bin Z, Guo-Rong L, Zu-Guang G, Sai-Ling H. An optical sensing system for the concentration of methane based on fiber Bragg gratings. Optoelectron Lett 2007;3:410-2.

[6] Renoirt JM, Caucheteur C, Mégret P, Debliquy M. Infrared radiation detector using a pair of fiber Bragg gratings. In: Proceedings of SPIE, vol. 7726; 2010. 772606-8.

[7] Caucheteur C, Renoirt JM, Debliquy M, Mégret P. Infrared radiation detection with matched fiber Bragg gratings. Photonics Technol Lett 2010;22:1732-4.

[8] Yüksel K, Mégret P, Wuilpart M. A quasi-distributed temperature sensor interrogated by optical frequency-domain reflectometer. Meas Sci Technol 2011;22:115204.

[9] Yüksel K, Wuilpart M, Moeyaert V, Mégret P. Novel monitoring technique for passive optical networks based on optical frequency domain reflectometry and fiber Bragg gratings. J Opt Commun Networking 2010;2:463-8.

[10] Aralt TT, Nilsen AR. Automatic fire detection in road traffic tunnels. Appl Opt 2008;24:75-83.

[11] Yüksel K, Wuilpart M, Moeyaert V, Mégret P. Complete analysis of multireflection and spectral-shadowing crosstalks in a quasi-distributed fibre sensor interrogated by OFDR. IEEE Sensors J 2011;17. 